\title{
An Imaging-based Autorefractor
}

\author{
Matteo Menolotto \\ Department of Biomedical Engineering \\ University of Strathclyde \\ Glasgow, UK \\ matteo.menolotto@tyndall.ie
}

\author{
Iain A. T. Livingstone \\ NHS Forth Valley \\ Falkirk Community Hospital \\ Falkirk, UK \\ iain.livingstone@nhs.net
}

\author{
Mario E. Giardini \\ Department of Biomedical Engineering \\ University of Strathclyde \\ Glasgow, UK \\ mario.giardini@strath.ac.uk
}

\begin{abstract}
Autorefraction consists of the automatic sensing of three parameters - spherical error, cylindrical error, slope of the principal meridian - that describe the deviation of the focusing properties of an ametropic eye with respect to an emmetropic state. Low-cost autorefractors would be highly desirable in resource-poor settings, for the stratification of patients between those who can be treated in the community and those who need to be referred to specialist care. In the present paper, we describe the implementation of an autorefractor based on projecting patterns onto the retina of an eye and observing the projected pattern through an ophthalmoscopic camera configuration coaxial with the projection path. Tunable optics in the coaxial path, combined with appropriate image processing, allows determination of the three parameters. The simplicity and performance of the setup, measured on an eye simulator, shows promise towards clinical use in the community. Further work is needed to confirm the performance in vivo.
\end{abstract}

Index Terms - refraction, vision tests, vision screening, optometry, community eye care, ophthalmoscopy

\section{INTRODUCTION}

In 2020, the World Health Organization estimated that 1 billion people have a visually impairment that could have been prevented [1]. The leading cause of visual impairment is uncorrected refractive error, which affects in excess of 900 million people [2]. Such visual impairment can be corrected with eye spectacles, effectively amongst the most highly costeffective eye care interventions. However, $90 \%$ of visually impaired people reside in low-income countries, where treatment provision is critically limited by the extremely low number of trained specialists with respect to the subtended population [3]. Yet, the patients requiring lower grades of correction can be successfully treated in the community through provision of ready-made spectacles, referring to specialist care only those with more severe levels of refractive error [4]. There is therefore a need for stratification within the community, by minimally trained non-specialist personnel, of the patients who can be treated locally with off-the-shelf spectacles, from those who need to be further referred to specialist care. This requires determination of the refractive error within the community.

Effectively refraction, the determination of the amount of uncorrected refractive error of an eye, consists of the sensing of three parameters that describe the deviation of the focusing properties of an ametropic eye, nominally looking at infinity,

This research has been funded by the Queen Elizabeth Diamond Jubilee Trust, project "Technology for Eye Health". with respect to an ideal focused (emmetropic) state. In particular, the error is described in terms of the composite sphericalcylindrical lens that needs to be placed in front of the eye to bring back the eye to focus onto the fovea - effectively, the spectacle lens that compensates the error. Such a lens is characterized by the focal length of the spherical and of the cylindrical (astigmatic) components, and by the angle of the principal meridian (axis) of the cylindrical component.

Traditionally, refraction is performed by a trained optometrist. The testing can either be objective, e.g. using retinoscopy [5], or subjective, whereby the subject under test makes binary choices of "better or worse" corrective lenses until an optimal visual acuity is attained. This remains the gold standard, and typical practice in the United Kingdom and, indeed, in most of the world. Devices that will automatically and, in principle, with little to no dependence on the operator skills, sense and measure refractive error (autorefractors) exist, and are used widely in both high street optometry and secondary care in the Western World. Autorefractors can broadly work relying on an image of the retina through the pupil (Scheiner, best-focus, knife-edge, image size, laser ray tracing, and Tscherning autorefractors), use the retina as a secondary image/radiation source (retinoscopic, optometer, and Hartmann-Shack autorefractors), or combine these principles in composite units [5]. These autorefractors are complex and expensive devices, typically costing in the 10,000 USD range and above for fixed units, and higher for portable handheld units. The high cost and fragility are hardly compatible with field/community use at scale. More recently, EyeNetra (Cambridge, MA, USA) has proposed commercially a phonebased manually assisted autorefraction tool [6]. The device is designed for self-use by the test subject, and implements a guided manual refraction procedure. Albeit less expensive than standard solutions, it relies on subjective feedback by the user on the device controls. It requires the subject to be able to operate the device controls reliably, and this requires training, and precludes the test on non-cooperating subjects, such young children or people with cognitive disabilities. Furthermore, the device is tied to a specific mobile phone model, and limited range of measurement spherical range -12 to +5.5 diopters, cylindrical range 0 to -7 diopters. The SVOne [7], a portable Hartmann-Shack wavefront aberrometer manufactured by Smart Vision Labs (New York, NY, USA), represents another smartphone-based adapter which shows promise when 
compared with conventional standards in normal adults and children outside the clinic setting.

In the recent past, we have shown that, with extremely simple and inexpensive hardware, it is possible to image the retina using a consumer-grade digital camera, such as a phone camera or a webcam [8]. We have shown the principle to be compatible with community screening, and successfully applied it for population screening in Kenya [9]. In the present paper, we describe the implementation of a variant on such configuration to sense and measure refractive error at a low cost with a fully automated protocol, in view of application for community screening by minimally trained personnel.

\section{AUtOREFRACTOR DESIGN}

Our autorefractor is represented in Fig. 1. A projection optics constituted of a DLP® LightCrafter Display 3010 EVM projector (Texas Instruments, TX, USA) focused at infinity is coaxially aligned through a Comar 40BN25 50\% plate beamsplitter (Comar Optics, United Kingdom) to a Logitech HD PRO Webcam C920 (Logitech SA, Switzerland) focused at infinity, both imaging on the retina of a Heine 000.33.011 retinoscopy eye simulator (Heine, Germany), thus implementing a direct ophthalmoscopy configuration [8] that views the pattern projected on the retina by the projector (2). An Optotune EL-16-40-TC-VIS-20D (Optotune, Switzerland) focus-tunable lens, whose focal length can be tuned between $-100 \mathrm{~mm}$ and $100 \mathrm{~mm}$ (-10 to 10 diopters) using an Optotune OEM lens driver, is inserted in the common optical path of the projector and camera. In turn, the retinoscopy eye simulator can be adjusted to simulate a refractive error between +7 and -7 diopters.

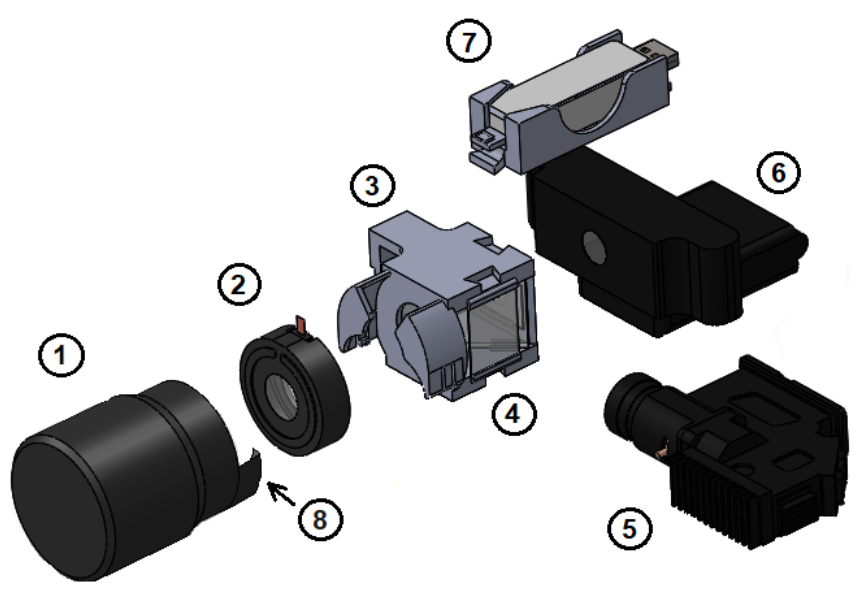

Fig. 1. (1) eye simulator; (2) focus-tunable lens; (3) 3D printed housing for the optics; (4) beam splitter and the circular polarizers; (5) DLP projector; (6) camera; (7) tunable lens driver; (8) lens holder.

As per refractive error simulation practice, a further +6 to -6 diopters cylindrical refractive error can be added onto the eye simulator by interposing cylindrical lenses from a standard optometry test (AWO-TLS266, Morton Medical, United Kingdom) set against the front surface of the eye simulator.
Two Comar 11WO50 laminated film circular polarizers on the projection and vision paths suppress stray reflections.

All components are assembled onto a standard aluminum optical breadboard (Thorlabs, NJ, USA), holding the optics and the circular polarizers in 3D printed custom housing (number (3) in Fig. 1) in order to minimize the distance between camera and eye simulator, which is $115 \mathrm{~mm}$, while the distance between the eye simulator and the tunable lens is kept to $55 \mathrm{~mm}$, thus maximizing the retinal field of view. A HP ProBook 430 G2 laptop computer (Hewlett-Packard, USA) running the MATLAB programming environment (The Mathworks, USA) controls the projector, the tunable lens and the camera. We note that MATLAB was used on a purely opportunistic basis, as available in our laboratory, and is immaterial for the correct working of the system, thus allowing the use of free or low-cost software.

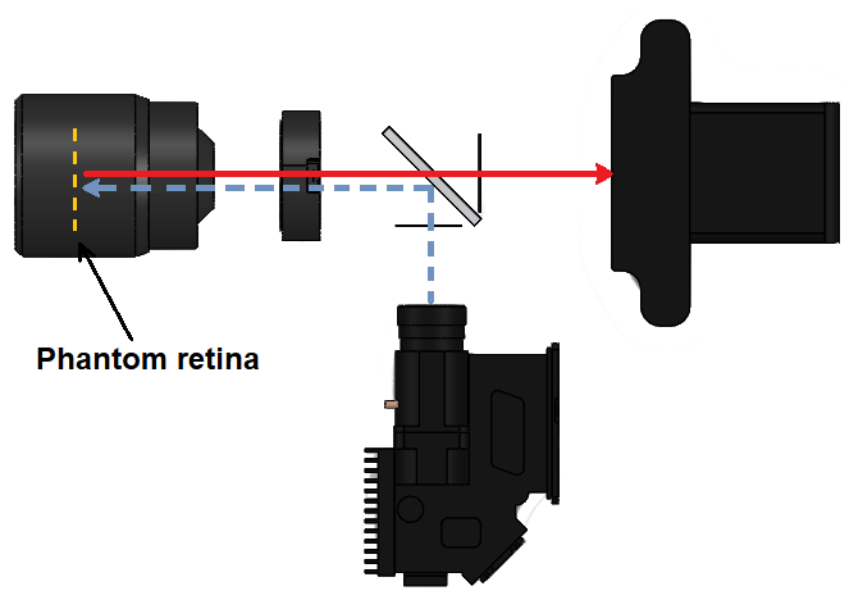

Fig. 2. Optical paths alignment. Blue, dotted line: projection path. Red, solid line: ophthalmoscopy path.

\section{WORKING PRINCIPLES}

In order to determine the spherical error, a grid pattern is projected onto the phantom retina Fig. 3). The size of the square composing the grid is set empirically to have visibility of at least 2-3 squares on a $3 \mathrm{~mm}$ pupil size on the eye simulator (corresponding to a portion of the image of 40 pixels at the resolution of $1280 \times 720$ ). All subsequent measurements are performed at such pupil size. The color of every pattern projected is green, as it maximises the contrast against the retinal tissues, simultaneously reducing the light penetration depth, thus enhancing the focus.

The camera visualizes the eye simulator retina through the eye pupil, eye lens and tunable lens, the beamsplitter and the circular polarizer (Fig. 3, left). In emmetropic conditions (absence of refractive errors), the image of the grid pattern projected onto the retina appears in focus when the tunable lens is set to zero diopters (no correction, infinite focal length). In the presence of refractive error, the viewing paths is defocused therefore also the pattern appears blurred. Along the same principle of viewing spectacles, such projection and 
viewing paths are brought back into focus when the tunable lens compensates the refractive error with a dioptric power of equal amplitude and opposite sign than the error itself. The spherical error is therefore determined from the dioptric power to be set on the tunable lens to yield the best focus of the grid as viewed on the retina by the camera.
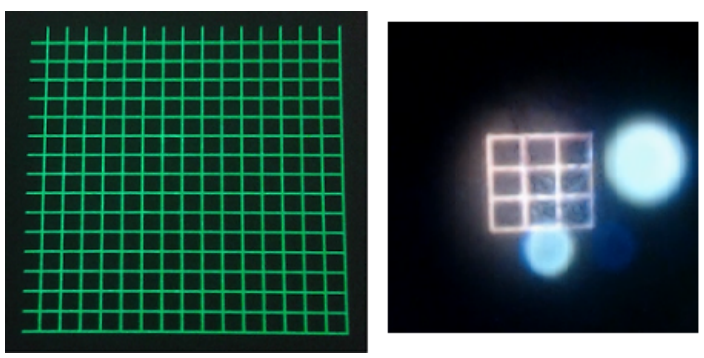

Fig. 3. Left image: grid pattern projected by the projection optics for the measurement of spherical error. Right image: square pattern projected onto the phantom retina of the eye simulator as seen by the camera.

In practice, in order to determine the refractive error set on the eye simulator, the dioptric power of the tunable lens is scanned in 0.25 diopters increments from +6 to -6 diopters, while images of the retina are acquired for each dioptric power increment. These images are then converted to grayscale, and the value of the Grey Level Local Variance (GLLV) is calculated for each image on a $15 \times 15$ pixel sliding window [10]. To obtain the GLLV, firstly the local variances within the window $\left(w_{\mathrm{x}}, w_{\mathrm{y}}\right)$ is evaluated, generating a new matrix of local variances values. Secondly, the standard deviation of such matrix is calculated. For each $k$ image $I$ of size $M \times N$, the local variance $(L V)$ at the point $(m, n)$, where $m=1, \ldots, M$ and $n=1, \ldots, N$, is given by:

$$
L V_{k}(m, n)=\frac{1}{w_{M} w_{N}} \sum_{i=1}^{w_{M}} \sum_{j=1}^{w_{N}}\left(I_{k}(m+i, n+j)-\bar{I}_{k}^{w}\right)^{2}
$$

where $\bar{I}_{k}{ }^{w}$ is the mean value of the $\mathrm{k}$ image within the sliding window. The final focus measure $(F M)$ is then given by the global variance of the $\mathrm{k}$ previously generated matrix, as:

$$
F M_{k}=\frac{1}{M N} \sum_{i=1}^{M} \sum_{j=1}^{N}\left(L V_{k}(m+i, n+j)-L \bar{V}_{k}\right)^{2}
$$

where $L V_{k}$ is the mean value of the local variance matrix of the $\mathrm{k}$ image.

This value is obtained for each image and stored in a vector during the process. The image with the higher FM corresponds to the optical setting that better compensates the spherical refractive error. Such setting is the optical power of the tunable lens, therefore the direct value of the corrective spectacles for the spherical error.

As for the cylindrical error determination, the tunable lens is set to best-focus as per the protocol above, and a square pattern (Fig. 4a) is projected onto the retina, of edge size adequate to have visibility of the full square (corresponding to a portion

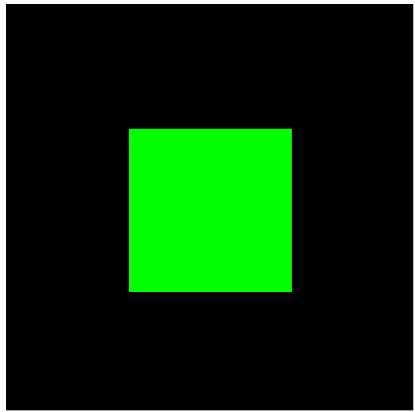

(a)

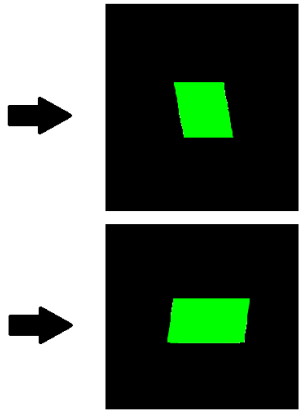

(b)
Fig. 4. (a) pattern to be projected on the retina; (b) Examples of distorted patterns, as seen by the ophthalmoscopic optical path, caused by cylindrical error, which causes the shrink in one dimension, and slope of principal meridian, which causes the skewing.

of the image of $\sim 50$ pixels at the resolution of $1280 \times 720$ ). A cylindrical lens, from a standard test set, is then interposed between the beamsplitter and the eye simulator. This generates a cylindrical deformation on the acquired image. The type of deformation is similar to the one shown in Fig. $4 \mathrm{~b}$.

The 2-dimensional Fast Fourier Transform (FFT) is calculated for the acquired image (Fig. 5, left), and the vertically and horizontally cross sections extracted (Fig. 5, right). The periodic spacing between the minima of the FFT in the two directions (non-zero due to the presence of noise and background in the image) relate to the compression of the square image in the two perpendicular directions due to the cylindrical error. Therefore, the ratio between the two spacings gives the ratio between the two sizes of the rectangle, which is correlated with the cylindrical error. To establish such correlation, a full characterization of such ration has been obtained for cylindrical error from -6 to 6 diopters in steps of 0.5 diopters, and the obtained calibration stored in a lookup table.
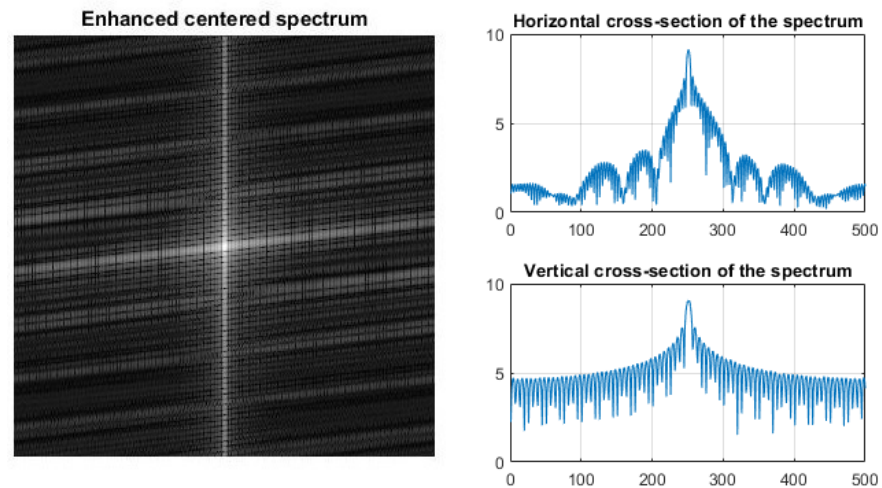

Fig. 5. (left) (left) Fast Fourier Transform of the square pattern, (right, top) horizontal and (right, bottom) vertical cross sections of FFT. The peaks correspond to the white lines in the FFT spectrum visualisation.

The system senses cylindrical refractive error as a different spacing in the two FFT cross sections, and reconstructs the value by referencing the ratio of the spacings to the lookup 
table. The direction of the principal meridian of the cylindrical error is visible in the FFT as direction of the maxima ridges of the FFT image (Fig. 5). Analytically, this angle $\alpha_{P M}$ is calculated by taking two vertical cross sections of the FFT image itself, at the positions $N / 2-t$ and $N / 2+t$, where $\mathrm{N}$ is the vertical size of the FFT image and $t$ is an arbitrary value between 1 and $M / 2-1$ (here is $M / 4$ ), finding the points in such vectors where the intensity is maximum (in a grayscale image the maximum is 1) and computing the trigonometric ratio between the vertical and the horizontal distance between these two points, as per the following relation:

$$
\alpha_{P M}=\tan ^{-1}\left[\left(y_{2}-y_{1}\right) /\left(x_{2}-x_{1}\right)\right]
$$

where $\left(x_{1}, y_{1}\right)$ and $\left(x_{2}, y_{2}\right)$ are the coordinates of the two maximum values of the two vertical cross sections of the FFT image.

\section{Results}

In Fig. 6 is reported the spherical refractive error measured on the eye simulator set to a nominal refractive error ranging from -6 to +6 diopters, in 1 diopter steps. 10 measurements have been taken for each refractive error value.

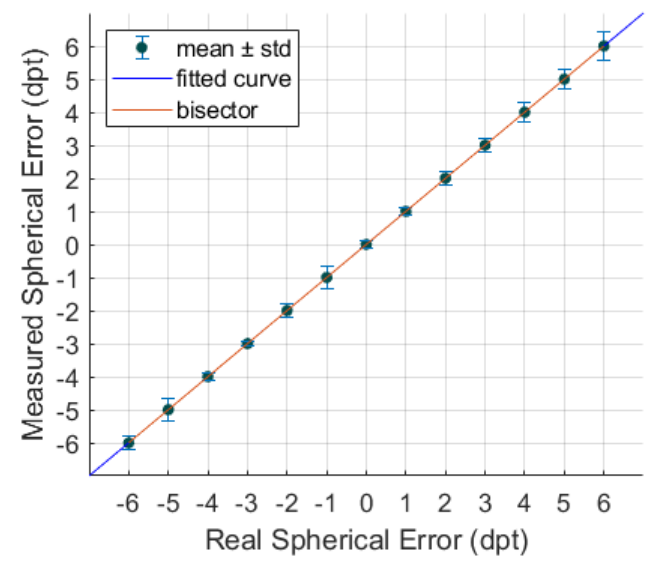

Fig. 6. Spherical error evaluation. The red bisector represents the ideal curve. The error bars represent the standard deviation.

We note that the uncertainty in the refractive error caused by the manual setting on the eye simulator is negligible with respect to the measurement error, and can therefore be neglected in the error analysis. Each measurement requires a total of $18 \mathrm{~s}$ of data acquisition, followed by $4 \mathrm{~s}$ of offline data processing. In Fig. 7 is reported the cylindrical refractive error measured on the eye simulator set at zero diopters spherical error, interposing a cylindrical lens ranging from -6 to 6 diopters in 1 diopter steps.

In Fig. 8 the direction of the principal meridian of the optical axis is reported, conventionally as angle from the direction perpendicular to the plane of the instrument ("vertical"), measured at a cylindrical refractive error of -4 diopters. Such direction was measured every $15^{\circ}$ step, between $0^{\circ}$ to $90^{\circ}$.

The maximum deviations over the real measurement and the standard deviations over the set of measurements for the

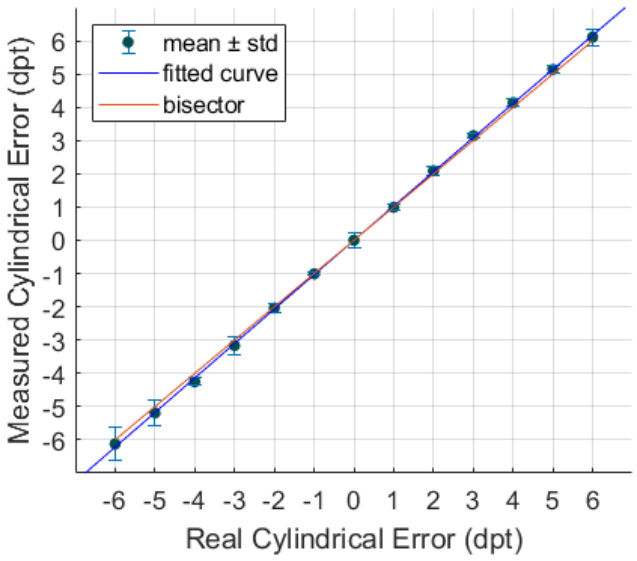

Fig. 7. Cylindrical error evaluation. The red bisector represents the ideal curve. The error bars represent the standard deviation.

spherical error, cylindrical error and slope of the principal meridian are 0.43 diopters, 0.50 diopters and $3.3^{\circ}$ respectively, with variances of 0.22 diopters, 0.19 diopters and $2.0^{\circ}$.

\section{Discussion}

Our results show that, on an eye simulator, the simple optical configuration employed yields an accuracy compatible with typical autorefractor performance [11]. The low standard deviation, which was expected, given the static test conditions, can be mostly ascribed to the only two movable components of the system, namely the focus-tunable lens and the eye simulator. The total cost of the components used is in the range of 2500 USD excluding the computer. However, plug-andplay programmable boards with DLP technology are currently available for less than 100 USD, effectively halving the costs and providing microcontrollers for embedded computing. At present, the DLP projector is used to project two static patterns, and the production cost of a final production-based unit can be therefore significantly reduced by employing a simple static mask-based projector.

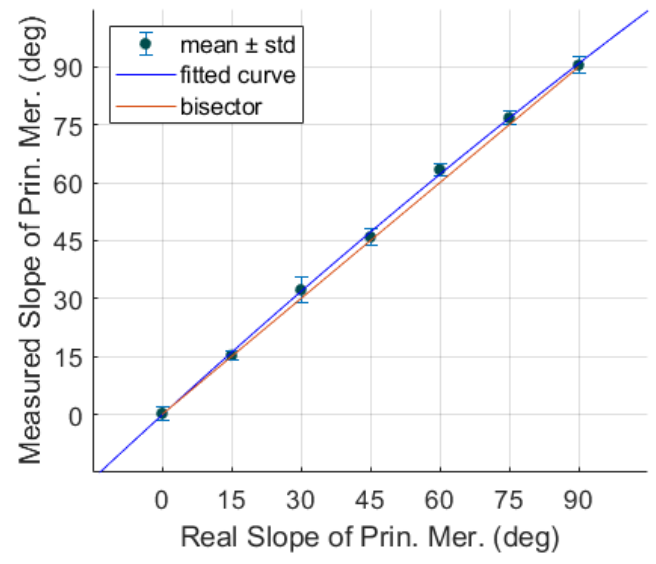

Fig. 8. Slope of principal meridian evaluation. The red bisector represents the ideal curve. The error bars represent the standard deviation. 
We note that, in the current setup, no hardware synchronization is provided between the camera and the tuning of the variable focus lens. As MATLAB does not provide for access to the frame synchronization of the camera, nor for real-time control of the lens driver, the synchronization between camera and lens is implemented through inefficient delay loops that ensure that the camera is acquiring only while the lens focus is stable. The data acquisition time is therefore severely suboptimal. In optimal conditions, considering that the sum of response and settling time of the focus-tunable lens is $\sim 50$ ms, synchronizing the lens focus adaptation with the dead time in the camera frames, even with a low-cost camera such as that employed in the study, which was set at 30 frames per second, the acquisition of the 57 frames captured in our protocol would require less than three seconds. This time can be further shortened by employing an extremum search algorithm, rather than a focus scan, e.g. implementing a traditional autofocus. Similarly, MATLAB has been employed for this study as a tradeoff between computational efficiency and software development flexibility. Improvements of two orders of magnitude or more in computing time have been reported when MATLAB is replaced by more conventional $\mathrm{C} / \mathrm{C}++$ code [12]. We can therefore assume that, in an optimal implementation, data acquisition and computing time do not constitute a bottleneck to the intended medical application.

As for all medical sensing, however, appropriate performance is defined by adequateness to the specific clinical requirements. In the process of refracting a patient's eyes to define the best pair of spectacles, the ultimate target lies in patient tolerance and acceptance of the optical prescription. It is well known that, even in ideal conditions of a clear eye lens and wide pupil, the readings given by an autorefractor show a higher variability in vivo than in an eye simulator [13]. This variability is caused by fluctuations in fixation and accommodation in a live patient, present even when the eye is stabilized pharmacologically, and the autorefractor itself does not contribute to this variability to any significant extent. Furthermore, patient acceptance is heavily affected by subjective factors, such as expectations and visual activity profile. This compounds to a difference between the prescription measured by automatic and subjective refraction, which can easily exceed one diopter even with highly stable autorefractors and expert subjective refraction operators [14]. This difference between subjective and automatic objective refraction is present even in patients in whom artificial intraocular lenses have been implanted, whose accommodation, by nature, does not fluctuate [15]. In any case, this clinical difference is much higher than the measurement error in our setup.

Indeed, one of the uses intended by the manufacturer for the eye simulator employed in this study is specifically the calibration and performance testing of autorefractors. We can therefore safely assume that, in our controlled simulator conditions, the measurements show promise of adequate performance in the field. However, in addition to the clinical factors mentioned above, autorefraction in vivo poses significant challenges due to the simultaneous combination of spherical and cylindrical error, and the additional presence of optical defects of real eyes, such as non-spherical deformations of the cornea (keratoconus causing irregular astigmatism) and lens opacity (cataract). These defects exhibit significant intersubject variability in their presentation, and are therefore not normally modelled in commercial eye simulators. Lens accommodation and alignment of the pupil to the projection path can instead be addressed by implementing an aiming fixation target in the data acquisition protocol (e.g. movable target that the operator can place on the wall behind), as standard practice in ophthalmoscopy. Future work will therefore be directed towards miniaturizing the autorefractor in a self-contained unit, in order to perform a full in-field pre-clinical testing for basic performance in vivo.

\section{CONCLUSIONS}

In this paper, we presented a new autorefractor concept that uses a projected pattern and automatic focus-tunable optics to objectively evaluate the refractive error of an eye simulator. The recent availability of low-cost tunable lenses has opened the way for the creation of new ophthalmic diagnostic tools, with potential benefits for population screening within hardto-rich communities, especially in resource-poor settings. This device, although in its proof of concept stage, has shown a viable way towards the development of inexpensive, easy to use and portable solution for clinical use in the field. More work is undergoing to reduce cost and size, for instance by replacing the projector with a simple collimated light source and laptop with an open-source single-board computer (e.g. Raspberry Pi). Further consultation with the medical field is required. Clinical tests will confirm the accuracy of the refractive error evaluation in vivo, as well as acceptability and reliability in the pediatric population, both crucial aspects for portable autorefractors [16].

\section{REFERENCES}

[1] (29 October 2020). Blindness and vision impairment. Available: https://www.who.int/news-room/fact-sheets/detail/blindness-and-visualimpairment

[2] R. R. A. Bourne et al., "Magnitude, temporal trends, and projections of the global prevalence of blindness and distance and near vision impairment: a systematic review and meta-analysis," The Lancet Global Health, vol. 5, no. 9, pp. e888-e897, 2017.

[3] A. Bastawrous and B. D. Hennig, "The global inverse care law: a distorted map of blindness," British Journal of Ophthalmology, vol. 96, no. 10 , pp. 1357-8, Oct 2012.

[4] T. S. Shane, O. Knight, W. Shi, J. C. Schiffman, E. C. Alfonso, and R. $\mathrm{K}$. Lee, "Treating uncorrected refractive error in adults in the developing world with autorefractors and ready-made spectacles," Clinical and Experimental Ophthalmology, vol. 39, no. 8, pp. 729-33, Nov 2011.

[5] M. Rosenfield and L. N, Optometry: Science, Techniques and Clinical Management. Elsevier Health Sciences, 2009.

[6] (29 October 2020). NETRA. Available: https://eyenetra.com/productnetra.html

[7] K. J. Ciuffreda and M. Rosenfield, "Evaluation of the SVOne: A Handheld, Smartphone-Based Autorefractor," Optometry and Vision Science, vol. 92, no. 12, pp. 1133-9, Dec 2015.

[8] M. E. Giardini et al., "A smartphone based ophthalmoscope," 36th Annual International Conference of the IEEE Engineering in Medicine and Biology Society (EMBC), vol. 2014, pp. 2177-80, 2014. 
[9] A. Bastawrous et al., "Clinical Validation of a Smartphone-Based Adapter for Optic Disc Imaging in Kenya," JAMA Ophthalmol, vol. 134, no. 2, pp. 151-8, Feb 2016.

[10] J. L. Pech-Pacheco, G. Cristobal, J. Chamorro-Martinez, and J. Fernandez-Valdivia, "Diatom autofocusing in brightfield microscopy: a comparative study," in 15th International Conference on Pattern Recognition (ICPR), 2000, vol. 3, pp. 314-317.

[11] S. Huffman. (29 October 2020). The accuracy of autorefractor measurements in young children. Available: http://www.aaopt.org/accuracyautorefractor-measurements-young-children

[12] T. Andrews, "Computation Time Comparison Between Matlab and C++ Using Launch Windows," BS thesis, Department of Aerospace Engineering, California Polytechnic State University.

[13] A. Rubin and W. F. Harris, "Refractive variation during autorefraction: multivariate distribution of refractive status," Optometry and Vision Science : Official Publication of the American Academy of Optometry, vol. 72, no. 6, pp. 403-10, Jun 1995.

[14] N. C. Strang, L. S. Gray, B. Winn, and J. R. Pugh, "Clinical evaluation of patient tolerance to autorefractor prescriptions," Clinical and Experimental Optometry, vol. 81, no. 3, pp. 112-118, May-Jun 1998.

[15] J. W. van der Linden, V. Vrijman, R. Al-Saady, I. J. van der Meulen, M. P. Mourits, and R. Lapid-Gortzak, "Autorefraction versus subjective refraction in a radially asymmetric multifocal intraocular lens," Acta Ophthalmologica, vol. 92, no. 8, pp. 764-8, Dec 2014.

[16] A. Samanta, A. Shetty, and P. C. Nelson, "Better one or two? A systematic review of portable automated refractors," Journal of Telemedicine and Telecare, Aug 102020. 